\title{
Errata
}

March 2003

Volume 111 | Number 5

\section{Rapid nontranscriptional activation of endothelial nitric oxide synthase mediates increased cerebral blood flow and stroke protection by corticosteroids}

Florian P. Limbourg, Zhihong Huang, Jean-Christophe Plumier, Tommaso Simoncini, Masayuki Fujioka, Jan Tuckermann, Günther Schütz, Michael A. Moskowitz, and James K. Liao

Original citation:J. Clin. Invest. 110:1729-1738 (2002). doi:10.1172/JCI200215481.

Citation for this erratum: J. Clin. Invest. 111:759 (2003). doi:10.1172/JCI200215481E1.

During the final stages of production, errors were introduced into Table 1. The corrected Table appears below. We regret the error and have provided the corresponding author with corrected reprints.

\section{Table 1}

Effects of high-dose corticosteroids on SV-129 mice

\begin{tabular}{|c|c|c|c|c|c|}
\hline & & Vehicle & Dex & Dex + RU & Dex + LY \\
\hline $\operatorname{MABP}(\mathrm{mm} \mathrm{Hg})$ & $\begin{array}{l}\text { Pre } \\
\text { MCAo } \\
\text { Post }\end{array}$ & $\begin{array}{c}106 \pm 112 \\
104 \pm 11 \\
99 \pm 8\end{array}$ & $\begin{array}{l}112 \pm 12 \\
107 \pm 11 \\
100 \pm 10\end{array}$ & $\begin{array}{c}109 \pm 11 \\
105 \pm 9 \\
98 \pm 10\end{array}$ & $\begin{array}{r}102 \pm 10 \\
98 \pm 12 \\
101 \pm 18\end{array}$ \\
\hline $\mathrm{CBF}(\%)$ & $\begin{array}{l}\text { Pre } \\
\text { MCAo } \\
\text { Post }\end{array}$ & $\begin{aligned} 100 & \pm 0 \\
10 & \pm 4 \\
103 & \pm 17\end{aligned}$ & $\begin{aligned} 100 & \pm 0 \\
8 & \pm 4 \\
107 & \pm 24\end{aligned}$ & $\begin{array}{c}100 \pm 0 \\
12 \pm 5 \\
102 \pm 19\end{array}$ & $\begin{array}{c}100 \pm 0 \\
15 \pm 8 \\
97 \pm 18\end{array}$ \\
\hline $\mathrm{pH}$ (arterial) & $\begin{array}{l}\text { Pre } \\
\text { MCAo } \\
\text { Post }\end{array}$ & $\begin{array}{l}7.32 \pm 0.04 \\
7.33 \pm 0.04 \\
7.33 \pm 0.02\end{array}$ & $\begin{array}{l}7.32 \pm 0.02 \\
7.32 \pm 0.03 \\
7.32 \pm 0.03\end{array}$ & $\begin{array}{l}7.37 \pm 0.03 \\
7.35 \pm 0.04 \\
7.36 \pm 0.04\end{array}$ & $\begin{array}{l}7.31 \pm 0.02 \\
7.35 \pm 0.02 \\
7.32 \pm 0.03\end{array}$ \\
\hline $\mathrm{paCO}_{2}(\mathrm{~mm} \mathrm{Hg})$ & $\begin{array}{l}\text { Pre } \\
\text { MCAo } \\
\text { Post }\end{array}$ & $\begin{array}{l}39.6 \pm 5.1 \\
40.4 \pm 6.9 \\
41.0 \pm 4.1\end{array}$ & $\begin{array}{l}41.4 \pm 4.2 \\
41.7 \pm 5.6 \\
43.4 \pm 2.7\end{array}$ & $\begin{array}{l}42.3 \pm 6.1 \\
41.5 \pm 4.9 \\
43.6 \pm 3.0\end{array}$ & $\begin{array}{l}42.3 \pm 5.6 \\
41.7 \pm 4.6 \\
39.4 \pm 4.5\end{array}$ \\
\hline $\mathrm{paO}_{2}(\mathrm{~mm} \mathrm{Hg})$ & $\begin{array}{l}\text { Pre } \\
\text { MCAo } \\
\text { Post }\end{array}$ & $\begin{array}{l}136 \pm 16 \\
143 \pm 12 \\
137 \pm 8\end{array}$ & $\begin{array}{l}136 \pm 19 \\
137 \pm 12 \\
128 \pm 10\end{array}$ & $\begin{array}{l}129 \pm 17 \\
145 \pm 15 \\
135 \pm 11\end{array}$ & $\begin{array}{l}124 \pm 13 \\
125 \pm 14 \\
128 \pm 15\end{array}$ \\
\hline $\mathrm{RT}\left({ }^{\circ} \mathrm{C}\right)$ & $\begin{array}{l}\text { Pre } \\
\text { MCAo } \\
\text { Post }\end{array}$ & $\begin{array}{l}36.9 \pm 0.2 \\
36.7 \pm 0.4 \\
36.5 \pm 0.3\end{array}$ & $\begin{array}{l}36.7 \pm 0.4 \\
36.8 \pm 0.4 \\
36.8 \pm 0.5\end{array}$ & $\begin{array}{l}37.2 \pm 0.3 \\
36.8 \pm 0.4 \\
36.8 \pm 0.4\end{array}$ & $\begin{array}{l}36.7 \pm 0.2 \\
37.1 \pm 0.4 \\
37.1 \pm 0.4\end{array}$ \\
\hline Mortality (\%) & & 10 & 8 & 10 & 9 \\
\hline \multicolumn{2}{|c|}{ Dex levels ( $\mu \mathrm{g} / \mathrm{dl}, 24$ hours) } & NA & $0.9 \pm 0.2$ & $0.7 \pm 0.1$ & $0.6 \pm 0.5$ \\
\hline
\end{tabular}

SV-129 mice were treated with dexamethasone $(20 \mathrm{mg} / \mathrm{kg}), \mathrm{RU} 486(200 \mathrm{mg} / \mathrm{kg})$, and LY294002 (5 mg/kg). Data are means \pm SD $(n=5-12)$. Dex, dexamethasone; RU, RU486; LY, LY294002; MABP, mean arterial blood pressure; Pre, before MCAo; Post, after MCAo; RT, rectal temperature; PaCO 2 , partial pressure arterial $\mathrm{CO}_{2} ; \mathrm{PaO}_{2}$, partial pressure arterial pressure $\mathrm{O}_{2} ; \mathrm{NA}$, not available.

\section{Paradoxical resistance to diet-induced obesity in UCP1-deficient mice}

Xiaotuan Liu, Martin Rossmeisl, Jennifer McClaine, and Leslie P. Kozak

Original citation: J. Clin. Invest. 111:399-407 (2003). doi:10.1172/JCI200315737.

Citation for this erratum: J. Clin. Invest. 111:759 (2003). doi:10.1172/JCI200315737E1.

During the final stages of production, errors were introduced into the author list. The corrected list with author affiliations appears below. We regret the error and have provided the corresponding author with corrected reprints.

Xiaotuan Liu, ${ }^{1}$ Martin Rossmeisl, ${ }^{1}$ Jennifer McClaine, ${ }^{1}$ Mark Riachi, ${ }^{2}$ Mary-Ellen Harper, ${ }^{2}$ and Leslie P. Kozak ${ }^{1}$

${ }^{1}$ Pennington Biomedical Research Center, Baton Rouge, Louisiana, USA

${ }^{2}$ Department of Biochemistry, Microbiology and Immunology, University of Ottawa, Ottawa, Canada 